\title{
Application of Fuzzy Theory to Plasma Data Analysis
}

\author{
Daizoh Nagahara, Zensho Yoshida, Nobuyuki Inoue \\ Department of Nuclear Engineering, Faculty of Engineering, \\ The University of Tokyo, Tokyo 113.
}

(Received July 13, 1989)

\begin{abstract}
To analyze correlations among time-series data in plasma experiments, the fuzzy theory has been applied. The method includes the standard spectral analysis as the simplest case, which is for statistically equilibrium fluctuations. A generalization made by the method extends to correlation analyses of various non-equilibrium fluctuations. Preliminary results of data analysis by the method has been obtained for the REPUTE-1 experiments.
\end{abstract}

Keywords:

data analysis, fuzzy theory,

\section{Introduction}

If fluctuations of measured values are statistically in equilibrium, i. e. , steady random processes, systematic methods can be applied. For example, spectral analyses are applicable for time series data, when the fluctuations are considered to be in statistical equilibria. Under the ergodic hypothesis, the ensemble average is can be replaced by the time average. Generally such a assumption does not hold. In plasma experiments, changes of some parameters during the life of a discharge are not steady statistical processes. For such phenomena, we want to find correlative relations among waveforms. A systematic scheme is required for quantifying the relation among waveforms.

Here we apply the fuzzy theory to evaluate some characteristics of waveforms. The fuzzy theory has been developed to analyze various fuzzy objects, for example, words spoken everyday, judgment, concept and so on 1),2). We develop a scheme for determining the correlative relation between the waveforms in plasma experiments. The scheme of this method consists of two main parts: (i) quantitatively evaluation for the shape of waveform, (ii) correlation analysis based on the evaluation.

In the next section, we give a short review of the fuzzy theory.

\section{Fundamentals of Fuzzy Theory}

A fuzzy set is a class of objects which is a continuum of grades of the membership. Such a set is characterized by a membership function, which assigns to each object a grade of the membership ranging between zero and unity. In this section, we shall discuss several basic definitions with respect to the fuzzy set.

Let $X$ be a space of objects with $x$ s;

$$
X=\{x\} .
$$


A fuzzy set $A$ over $X$ is characterized by a membership function $f_{A}(x)$. Here $f_{A}(x)$ is valued in the interval $[0,1]$. The value of $f_{A}(x)$ at $x$ represents the grade of the membership of $x$ in $A$ :

$$
f_{A}: X \rightarrow[0,1]
$$

Therefore the value of $f_{A}(x)$ close to unity implies that the grade of the membership of $x$ in $A$ is high.

For example, let $X$ be the real number $\mathbf{R}^{1}$ and let $A$ be a fuzzy set of numbers which is close to 3 . Then, representative values of the membership function $f_{A}(x)$ are, for example,

$$
\begin{gathered}
f_{A}(0)=0.1 ; f_{A}(1)=0.5 ; f_{A}(2)=0.8 ; \\
f_{A}(3)=1.0 ; f_{A}(4)=0.8 ; f_{A}(5)=0.5 ; \\
f_{A}(6)=0.1 .
\end{gathered}
$$

We start with several basic definitions with respect to fuzzy sets. The definitions are obviously conceptual extensions of the corresponding definitions for ordinary sets (often called crisp sets). Note that a crisp set corresponds to a fuzzy set, when we set $f_{A}(x)$ to take only two values, zero and unity, according to whether $x$ belong to $A$ or not. In the following discussions, the usual notations for crisp sets are used:

$$
\begin{array}{ll}
A^{c} & : \text { complement of a set } A, \\
\Phi & : \text { empty set, } \\
A \cup B & : \text { union of two sets } A \text { and } B, \\
A \cap B & : \text { intersection of two sets } A \text { and } B, \\
A \subset B & : \text { containment of a set } A \text { in a set } B .
\end{array}
$$

The relation of inclusion $A \subset B$ for two fuzzy sets $A$ and $B$ is defined by,

$$
f_{A}(x) \leq f_{B}(x) \text {, for all } x \in X
$$

Obviously the equivalence $A=B$ implies

$$
f_{A}(x)=f_{B}(x) \text {, for all } x \in X .
$$

The complement $A^{c}$ of a fuzzy set $A$ is defined by the membership function,

$$
f_{A} c(x)=1-f_{A}(x) \text {, for all } x \in X .
$$

Then the union and the intersection of two fuzzy sets $A$ and $B$ are respectively defined by,

$$
\begin{aligned}
& f_{A \cup B}(x)=f_{A}(x) \uparrow f_{B}(x), \text { for all } x \in X . \\
& f_{A \cap B}(x)=f_{A}(x) \downarrow f_{B}(x), \text { for all } x \in X .
\end{aligned}
$$

where the notations $\uparrow$ and $\downarrow$ mean, 


$$
\begin{aligned}
& \xi \uparrow \eta=\max [\xi, \eta], \\
& \xi \downarrow \eta=\min [\xi, \eta],
\end{aligned}
$$

respectively.

These operations conserve almost all the properties of those for crisp sets. However, the law of the excluded middle and the law of the contradiction in crisp sets are not satisfied, i.e. ,

$$
\begin{aligned}
& A \cup A^{c} \neq X, \\
& A \cup A^{c} \neq \Phi,
\end{aligned}
$$

where $X$ denotes the whole set.

As in the case of crisp sets, De Morgan's law and the law of the double negative are satisfied, i. e. , for two fuzzy sets $A$ and $B$,

$$
\begin{aligned}
& (A \cup B)^{c}=A^{c} \cup B^{c}, \\
& (A \cap B)^{c}=A^{c} \cap B^{c},
\end{aligned}
$$

and,

$$
\left(A^{c}\right)^{c}=A \text {. }
$$

\section{Method of Evaluation}

In this section, we describe how shapes of waveforms is quantified, applying the fuzzy theory. Using the quantified values, a scheme of the correlation analysis is developed.

\section{( i ) Classification of Waveform}

First, we observe waveforms to extract characteristics to specify the data. Here the characterization is in general a fuzzy procedure. Standards for the classification of the shapes of waveforms should be made clear.

(ii) Modeling for Waveform

Experientially, well-grounded notions for the property are needed to clear up, and undeniable interpretations based on this premise should be utilized to construct a model. The model should also be a simple expression of the essence of the property.

(iii) Definition of Evaluation Function

Constructing a model for a waveform, the next procedure is to define a evaluation function that is a membership function. We have defined,

$$
h(f) \equiv e^{-\alpha N(f)},
$$

where

$$
N(f) \equiv \int_{T_{1}}^{T_{2}}\left[f(t)-f_{0}(t)\right]^{2} d t / \int_{T_{1}}^{T_{2}} f_{0}^{2}(t) d t .
$$

In Eq. (16), $f(t)$ and $f_{0}(t)$ denote a experimental waveform and the model waveform, respectively. The interval $\left[T_{1}, T_{2}\right]$ of integration corresponds to one that the model is defined. The value of $N$ expresses the degree of the discrepancy between the model waveform and the experimental waveform. 
(iv) Method of correlative analysis

Next, using these values, we develop the correlation analysis.

Let $h(x)$ and $g(y)$ be the evaluation functions defined by Eq. (15). Then the degree of the correlation between $h(x)$ and $g(y)$, which we denote by $\pi(x ; y)$, is defined by,

$$
\pi(x ; y) \equiv \min [h(x), g(y)],
$$

for every $x \quad X, y \quad Y$. Equation (17) is calculated for every combination $(x, y)$ sampled from the data set. Both the values of $h(x)$ and $g(y)$ which are close to unity imply that the value of $\pi(x ; y)$ is close to unity. Therefore the greater value of $\pi(x ; y)$ means a stronger correlation. On the other hand, if either the value of $h(x)$ or that of $g(y)$ is small, the estimated value of $\pi(x ; y)$ is small. This means that the correlation is weak.

Let $\pi_{j}(x ; y)$ denote the value of $\pi(x ; y)$ for the $j$ th sample. The second procedure is to calculate the following quantity:

$$
\operatorname{cor}(x ; y) \equiv \frac{1}{M} \sum_{j=1}^{m} \pi_{j}(x ; y),
$$

where

$$
M \equiv \sum_{j=1}^{m} \max [h(x), g(y)] .
$$

The value $\operatorname{cor}(x ; y)$ is calculated for every $x \in X, y \in Y$. The notations of $m$ and $j$ stand for the whole sum of samples and the $j$-th sample, respectively, and $M$ is for the normalization. When the correlation is strong, the value of $\operatorname{cor}(x ; y)$ is close to unity.

\section{Application for REPUTE-1 Data Analysis}

The scheme described in the previous section has been applied to the correlation analysis for the REPUTE-1 data base ${ }^{3)}$. Here we describe a result of the analysis of the relation between the plasma current and the electron density.

The models for them are shown in Fig. 1 and Fig. 2. Figure 1 shows the model for the

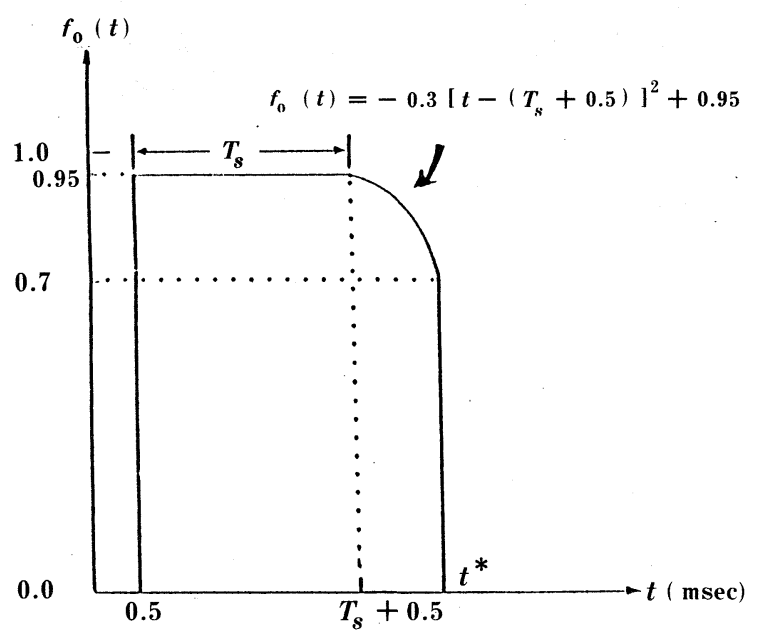

Fig. 1: A model of the waveform for evaluation of the sustainment time of the plasma current in ULQ discharge. 


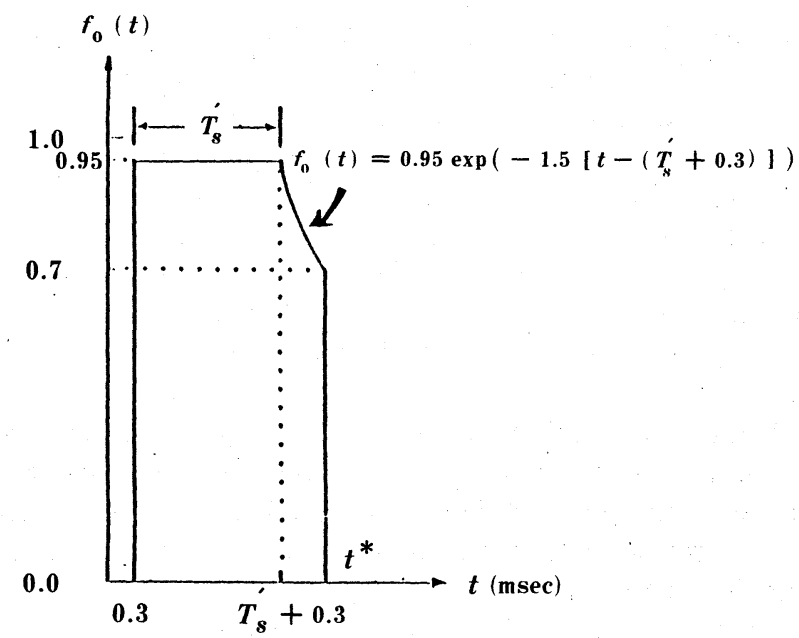

Fig. 2: A model of the waveform for evaluation of the sustainment time of the electron density in ULQ discharge.
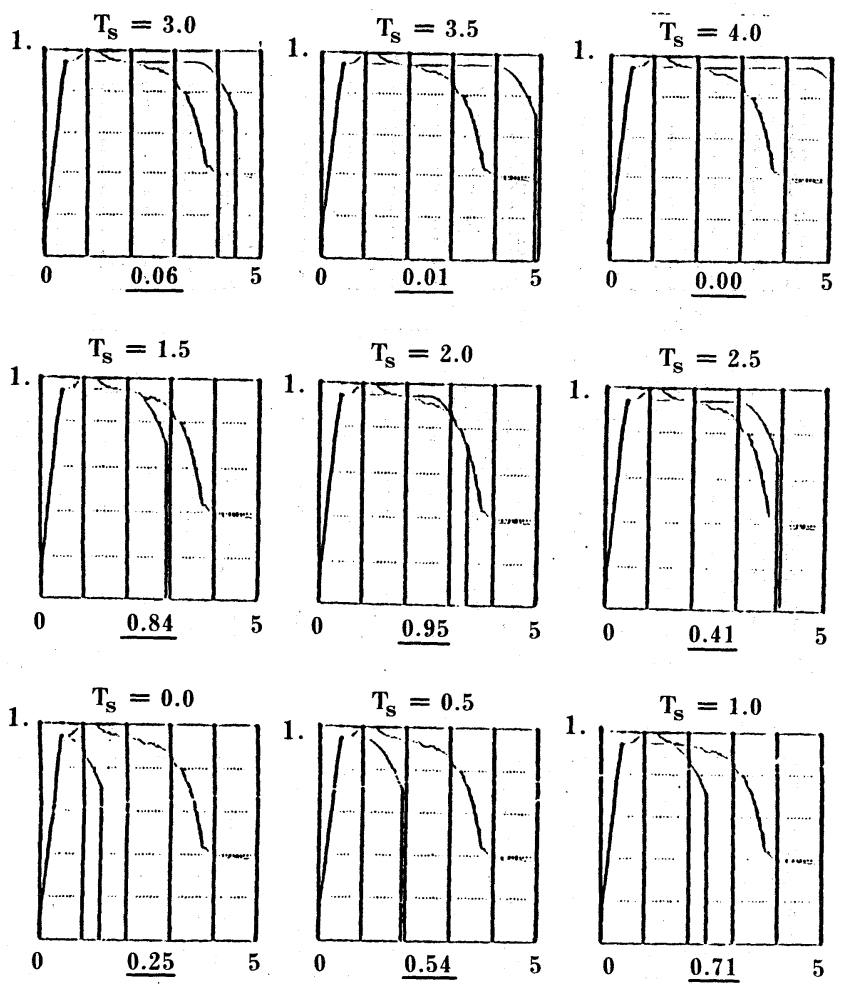

Fig. 3: Application of the model Fig. 1 to the plasma current in REPUTE-1. 
sustainment time of the plasma current in ULQ discharge. The interval of integration corresponds to $\left[0.5, t^{*}\right]$, which is experientially determined. Let the characteristic parameter $T_{s}$, which will be used to the correlative analysis, stand for the sustainment time in a sense. Figure 2, similarly, shows the model for the sustainment time of the electron density in ULQ discharge. The interval of integration corresponds to $\left[0.3, t^{*}\right]$.

Figure 3 shows the result of the application of the model Fig. 1 to the plasma current in REPUTE-1, using Eqs. (15) and (16). In Eq. (15) the value of $\alpha$ is chosen as 50.

Figure 4 shows the result of the application of the model Fig. 2 to the electron density for the same shot in Fig. 3, using Eqs. (15) and (16). In Eq. (15) the value of $\alpha$ is chosen as 14 .

In Eq. (17) the evaluation function $h(x)$ and $g(y)$ correspond to estimating the sustainment time of the plasma current and that of the electron density, respectively. Then the dependent variable $x$ for the function $h$ corresponds to the sustainment time for the current $T_{s}$ itself, and $y$ for $g$ corresponds to that for the electron density $T_{s}^{\prime}$ itself, i.e., in the example of Fig. 3 and Fig. 4 ,

$$
\begin{aligned}
& X=T_{s}=\{0.0,0.5,1.0,1.5,2.0,2.5,3.0,3.5,4.0\}, \\
& X=T_{s}^{\prime}=\{0.0,0.1,0.2,0.3,0.4,0.5,0.6,0.7,0.8\},
\end{aligned}
$$

respectively. Note that both $h(x)$ and $g(y)$ are not continuous functions, but discrete quantities.
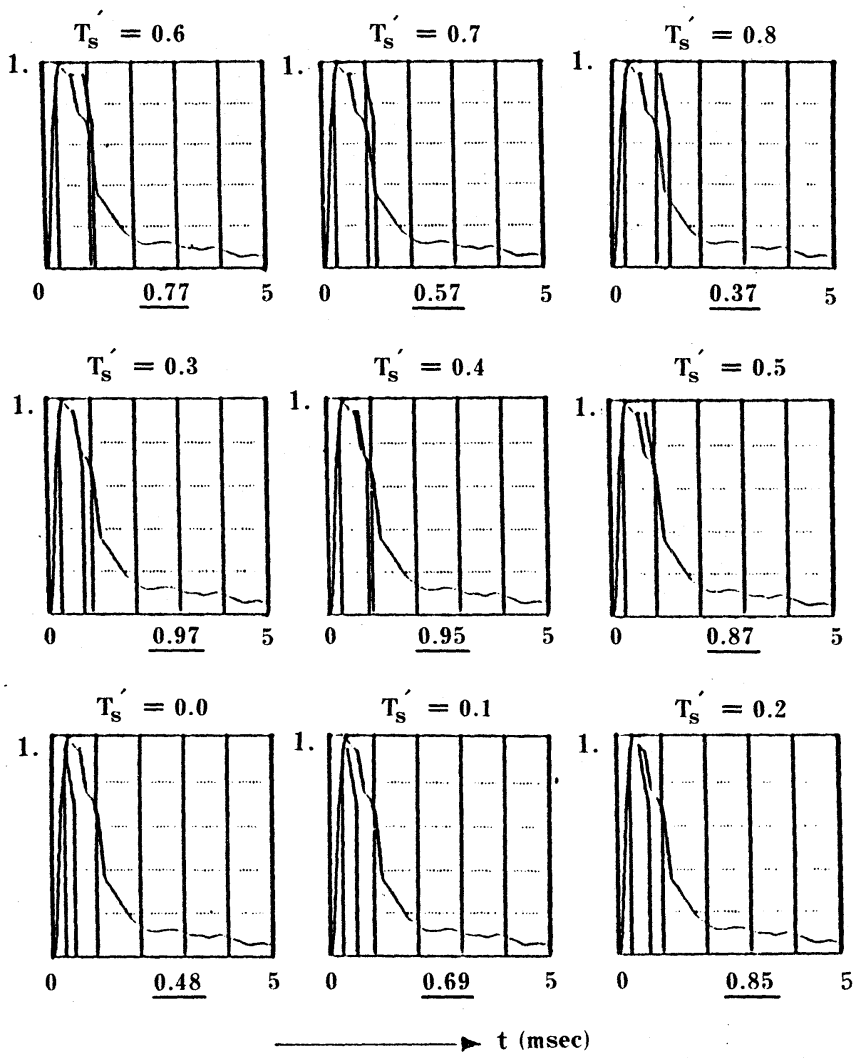

Fig. 4: Application of the model Fig. 2 to the electron density for the same shot in Fig. 3. 
Using the results obtained, the correlation has been analyzed according to the procedure described in the previous section. The result is shown in Table I . Here we enhance the steepness of the evaluation functions $h$ and $g$. We modify the values of $h$ less than 0.7 to 0 , and $g$ less than 0.8 to 0 . This procedure have been done for simplification of calculations. From Table $I$, the prediction that $T_{s} \sim 2.0$ and $T_{s}^{\prime} \sim 0.25$ may be deduced.

The last procedure is to analyze the correlation between two parameters. The result for the total shot of 100 is shown in Table II . In addition, the result is three-dimensionally shown in Fig. 5, so as to be easy to evaluate. Observing this figure, the positive correlative relation between them is estimated.

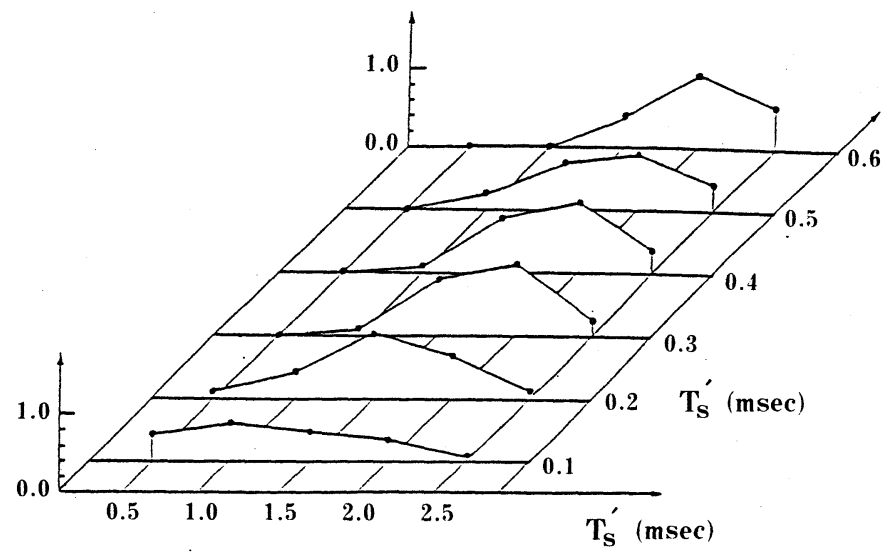

Fig. 5: Three-dimensional illustration of the degree of the correlation $\pi(x ; y)$.

Table I: Values of $\pi\left(T_{s} ; T_{s}^{\prime}\right)$ defined by Eq. (17).

\begin{tabular}{|c|c|c|c|c|c|c|}
\cline { 2 - 7 } \multicolumn{2}{c|}{} & \multicolumn{5}{|c|}{ Ts } \\
\cline { 2 - 7 } & 0.5 & 1.0 & 1.5 & 2.0 & 2.5 \\
\hline \multirow{4}{*}{ Ts' } & 0.1 & 0.00 & 0.00 & 0.00 & 0.85 & 0.00 \\
\cline { 2 - 7 } & 0.2 & 0.00 & 0.71 & 0.84 & 0.95 & 0.00 \\
\cline { 2 - 7 } & 0.3 & 0.00 & 0.71 & 0.84 & 0.95 & 0.00 \\
\cline { 2 - 7 } & 0.4 & 0.00 & 0.71 & 0.84 & 0.87 & 0.00 \\
\cline { 2 - 7 } & 0.5 & 0.00 & 0.71 & 0.84 & 0.00 & 0.00 \\
\hline & 0.6 & 0.00 & 0.00 & 0.00 & 0.00 & 0.00 \\
\hline
\end{tabular}

Table II: Values of cor $\left(T_{s} ; T_{s}^{\prime}\right)$ calculated by data from 100 shots, using Eqs. (18) and (19).

\begin{tabular}{|c|c|c|c|c|c|c|}
\cline { 2 - 7 } \multicolumn{1}{c|}{} & \multicolumn{5}{|c|}{ Ts } \\
\cline { 2 - 7 } & 0.5 & 1.0 & 1.5 & 2.0 & 2.5 \\
\hline \multirow{4}{*}{$T^{\prime}$} & 0.1 & 0.35 & 0.50 & 0.39 & 0.26 & 0.04 \\
\cline { 2 - 7 } & 0.2 & 0.09 & 0.33 & 0.82 & 0.54 & 0.05 \\
\cline { 2 - 7 } & 0.3 & 0.00 & 0.06 & 0.76 & 0.91 & 0.20 \\
\cline { 2 - 7 } & 0.4 & 0.00 & 0.06 & 0.71 & 0.89 & 0.28 \\
\hline & 0.5 & 0.00 & 0.19 & 0.59 & 0.70 & 0.33 \\
\hline & 0.6 & 0.00 & 0.00 & 0.40 & 0.96 & 0.51 \\
\hline
\end{tabular}

\section{Conclusion}

We developed a method of the correlation analysis of data whose fluctuation is not in a statistical equilibrium. The outline of this scheme is summarized as follows:

(i ) Classifying waveforms, we extract characteristic properties.

(ii) We define a standard waveform. 


\section{核融合研究 第62巻第 2 号 1989 年 8 月}

(iii ) The evaluation function, which describes the distance between a experimental waveform and the model one, is calculated.

(iv) Using Eqs. (17), (18) and (19), We estimate the correlative relation.

Applying this scheme to the experimental data in ULQ plasmas on REPUTE-1, the

correlative relation between two parameters has been quantified.

\section{REFERENCES}

1) L. A. Zadeh : Fuzzy Sets and Applications (Wiley-Interscience, New York, 1987).

2) L. A. Zadeh : Fuzzy Sets, Information and Control 8 (1965) 338.

3) Y. Iwata, K. Hattori, Z. Yoshida, and N. Inoue : Kakuyugo-Kenkyu 57 (1987) 441 [in Japanese]. 